ANNALES

POLONICI MATHEMATICI

$94.1(2008)$

\title{
Foliations by surfaces of a peculiar class
}

\author{
by ADAM BARTOSZEK (Łódź) and \\ PAWEe Walczak (Łódź and Warszawa)
}

\begin{abstract}
We classify surfaces in 3-dimensional space forms which have all the local conformal invariants constant and show that compact 3-manifolds of nonzero constant sectional curvature admit no foliations by such surfaces.
\end{abstract}

Introduction. It is well known that there exist several obstructions to the existence (on given Riemannian manifolds) of foliations with all the leaves satisfying some geometric properties. For example, by purely topological reasons, there exist no codimension-one totally geodesic foliations of round spheres and, by rather dynamical arguments, no such foliations exist on compact manifolds of negative sectional curvature (in any dimension). Also, there exist no codimension-one totally umbilical foliations of compact manifolds of negative Ricci curvature; in fact, all the foliations of such manifolds are, in a sense, far from being umbilical [LW]. Umbilicity is a conformal invariant: if $p$ is an umbilical point of a hypersurface $N$ of a Riemannian manifold $(M, g)$, and $\widetilde{g}=e^{2 \psi} g$ is a Riemannian metric conformally equivalent to $g$, then $p$ is umbilical for $N$ on $(M, \widetilde{g})$. This is why Remi Langevin and the second author were searching for other conformally invariant properties providing obstructions to existence of foliations enjoying these properties and have shown $[\mathrm{LW}]$ that compact 3-dimensional hyperbolic manifolds admit no foliations by Dupin cyclides which can be characterized by vanishing of both conformal principal curvatures (cf. Section 1 below). Here, we go one step further: after describing (following [CSW], [Fi] and [Br]) in Section 1 local conformal invariants of surfaces and classifying in Section 2 surfaces with all the conformal invariants constant, we prove in Section 3 our main result which says that compact hyperbolic 3-manifolds admit no CCI-foliations, that is, foliations by surfaces with constant conformal invariants.

2000 Mathematics Subject Classification: 53C12, 53A30.

Key words and phrases: foliation, surface, local conformal invariants. 
1. Local conformal invariants. Let $S$ be an oriented surface in $\mathbb{R}^{3}$. Assume that $S$ is umbilic free, that is, the principal curvatures $k_{1}(x)$ and $k_{2}(x)$ of $S$ are different at each point $x$ of $S$. Let $X_{1}$ and $X_{2}$ be unit vector fields tangent to the curvature lines corresponding to, respectively, $k_{1}$ and $k_{2}$. Throughout the paper, we assume that $k_{1}>k_{2}$. Put $\mu=$ $\left(k_{1}-k_{2}\right) / 2$. For more than 100 years, it has been known ([Tr], see also $[\mathrm{CSW}])$ that the vector fields $\xi_{i}=X_{i} / \mu$ and the coefficients $\theta_{i}$ $(i=1,2)$ in

$$
\left[\xi_{1}, \xi_{2}\right]=-\frac{1}{2}\left(\theta_{2} \xi_{1}+\theta_{1} \xi_{2}\right)
$$

are invariant under an arbitrary (orientation preserving) conformal transformation of $\mathbb{R}^{3}$. (In fact, they are invariant under an arbitrary conformal change of the Riemannian metric on the ambient space.) Elementary calculation involving the Codazzi equations shows that

$$
\theta_{1}=\frac{1}{\mu^{2}} \cdot X_{1}\left(k_{1}\right) \text { and } \theta_{2}=\frac{1}{\mu^{2}} \cdot X_{2}\left(k_{2}\right) .
$$

The quantities $\theta_{i}(i=1,2)$ are called the conformal principal curvatures of $S$.

Another conformally invariant scalar quantity $\Psi$ can be derived from the derivation of Bryant's (see $[\mathrm{Br}]$ ) conformal Gauss map $\beta$ :

$$
\begin{aligned}
\frac{1}{2}\left(\left\langle\xi_{1}\left(\xi_{1}(\beta)\right), \xi_{1}\left(\xi_{1}(\beta)\right)\right\rangle-\right. & \left\langle\xi_{2}\left(\xi_{2}(\beta)\right), \xi_{2}\left(\xi_{2}(\beta)\right)\right\rangle \\
& \left.-\left\langle\xi_{1}\left(\xi_{1}(\beta)\right), \xi_{2}(\beta)\right\rangle^{2}+\left\langle\xi_{2}\left(\xi_{2}(\beta)\right), \xi_{1}(\beta)\right\rangle\right) \\
= & \Psi-\frac{1}{2}\left(\theta_{1}^{2}-\theta_{2}^{2}+\xi_{1}\left(\theta_{1}\right)+\xi_{2}\left(\theta_{2}\right)\right) .
\end{aligned}
$$

Note that both sides of the above equality are equal to

$$
\frac{1}{\mu^{3}}\left(\Delta H+2 \mu^{2} H\right)
$$

where $H$ is the mean curvature of $S$, and $\Delta$ is the Laplace operator on $S$ equipped with the Riemannian metric induced from the ambient space. Moreover, this quantity appears in the Euler-Lagrange equation for the Willmore functional

$$
\int_{S} \mu^{2} d \text { area. }
$$

The vector fields $\xi_{1}, \xi_{2}$ (or the dual 1-forms $\omega_{1}, \omega_{2}$ ) together with the quantities $\theta_{1}, \theta_{2}$ and $\Psi$ generate all the local conformal invariants for surfaces and determine a surface up to conformal transformations of $\mathbb{R}^{3}$ ([Fi], see again $[\mathrm{CSW}])$. 
Define $5 \times 5$ matrices $A_{1}$ and $A_{2}$ by

$$
\begin{aligned}
A_{1} & =\left(\begin{array}{ccccc}
\theta_{1} / 2 & -(1+\Psi) / 2 & b / 2 & \theta_{1} / 2 & 0 \\
1 & 0 & 0 & -1 & (1+\Psi) / 2 \\
0 & 0 & 0 & 0 & -b / 2 \\
0 & 1 & 0 & 0 & -\theta_{1} / 2 \\
0 & -1 & 0 & 0 & -\theta_{1} / 2
\end{array}\right), \\
A_{2} & =\left(\begin{array}{ccccc}
-\theta_{2} / 2 & -c / 2 & -(1-\Psi) / 2 & \theta_{2} / 2 & 0 \\
0 & 0 & 0 & 0 & c / 2 \\
1 & 0 & 0 & 1 & (1-\Psi) / 2 \\
0 & 0 & -1 & 0 & -\theta_{2} / 2 \\
0 & 0 & -1 & 0 & \theta_{2} / 2
\end{array}\right),
\end{aligned}
$$

where $b=-\theta_{1} \theta_{2}+\xi_{2}\left(\theta_{1}\right)$ and $c=\theta_{1} \theta_{2}+\xi_{1}\left(\theta_{2}\right)$.

Given, on a simply connected domain $U \subset \mathbb{R}^{2}$, linearly independent 1forms $\omega_{1}$ and $\omega_{2}$ and smooth functions $\theta_{1}, \theta_{2}$ and $\Psi$ for which the matrix valued 1 -form

$$
\omega=A_{1} \omega_{1}+A_{2} \omega_{2}
$$

satisfies the structural equation

$$
d \omega+\frac{1}{2}[\omega, \omega]=0,
$$

there exists an immersion $\iota: U \rightarrow \mathbb{R}^{3}$ for which $S=\iota(U)$ realizes these forms and functions as local conformal invariants.

2. Integrability. Let us assume that $\theta_{1}$ and $\theta_{2}$ are constant, $\omega_{1}=$ $f_{1} d x_{1}$ and $\omega_{2}=f_{2} d x_{2}$, where $\left(x_{1}, x_{2}\right)$ are the Cartesian coordinates on the plane $\mathbb{R}^{2}$. Then the quantities $b=-\theta_{1} \theta_{2}$ and $c=\theta_{1} \theta_{2}$ in (1) and (2) are also constant, and therefore the only nonconstant entries of the matrices $A_{1}$ and $A_{2}$ are those involving $\Psi$. The structural equation (4) can be written in the form

$$
-f_{1} \frac{\partial A_{1}}{\partial x_{2}}-\frac{\partial f_{1}}{\partial x_{2}} A_{1}+f_{2} \frac{\partial A_{2}}{\partial x_{1}}+\frac{\partial f_{2}}{\partial x_{1}} A_{2}+f_{1} f_{2}\left[A_{1}, A_{2}\right]=0
$$

and reduces in the case of constant conformal principal curvatures to the system of four partial differential equations

$$
\begin{gathered}
-f_{1} \frac{\partial \Psi}{\partial x_{2}}+\frac{\partial f_{1}}{\partial x_{2}}(1+\Psi)-\theta_{1} \theta_{2} \frac{\partial f_{2}}{\partial x_{1}}-\frac{1}{2} f_{1} f_{2}\left(\theta_{1}^{2} \theta_{2}+3 \theta_{2}+\theta_{2} \Psi\right)=0, \\
\theta_{1} \theta_{2} \frac{\partial f_{1}}{\partial x_{2}}-f_{2} \frac{\partial \Psi}{\partial x_{1}}-\frac{\partial f_{2}}{\partial x_{1}}(1-\Psi)-\frac{1}{2} f_{1} f_{2}\left(\theta_{1} \theta_{2}^{2}+3 \theta_{1}-\theta_{1} \Psi\right)=0,
\end{gathered}
$$




$$
\frac{\partial f_{1}}{\partial x_{2}}=-\frac{1}{2} f_{1} f_{2} \theta_{2} \quad \text { and } \quad \frac{\partial f_{2}}{\partial x_{1}}=\frac{1}{2} f_{1} f_{2} \theta_{1} .
$$

These four equations arise from the matrix equation (5) by considering respectively entries $(1,2),(1,3),(2,1)$ and $(3,1)$. These equations imply the following ones:

$$
\begin{aligned}
& \frac{\partial \Psi}{\partial x_{1}}+2 f_{1} \theta_{1}-f_{1} \theta_{1} \Psi+f_{1} \theta_{1} \theta_{2}^{2}=0 \\
& \frac{\partial \Psi}{\partial x_{2}}+2 f_{2} \theta_{2}+f_{2} \theta_{2} \Psi+f_{1} \theta_{1}^{2} \theta_{2}=0
\end{aligned}
$$

which - after differentiating and comparing the derivatives $\partial^{2} \Psi / \partial x_{1} \partial x_{2}$ and $\partial^{2} \Psi / \partial x_{2} \partial x_{1}$ - yield the identity

$$
\theta_{1} \theta_{2}\left(1+\frac{1}{2} \theta_{2}^{2}+\frac{1}{2} \theta_{2}^{2}\right)=0 .
$$

This shows the following.

Proposition 1. Any surface $S \subset \mathbb{R}^{3}$ with constant conformal principal curvatures has at least one of these curvatures equal to zero.

Compact surfaces with both conformal principal curvatures equal to zero are well known: they are so called Dupin cyclides, that is, conformal images of tori of revolution. Other surfaces with vanishing conformal principal curvatures arise as conformal images of a cylinder or cone (of revolution).

Assume now that $\theta_{1}=0$ while $\theta_{2}=c$ is an arbitrary nonzero constant. Then $\partial f_{2} / \partial x_{1} \equiv 0$ and $f_{2}$ is a function of $x_{2}$ only. Since $\partial\left(\log f_{1}\right) / \partial x_{2}$ $=-\frac{1}{2} c f_{2}$,

$$
f_{1}\left(x_{1}, x_{2}\right)=C_{1}\left(x_{1}\right) \cdot e^{-\frac{1}{2} c \int_{0}^{x_{2}} f_{2}(t) d t},
$$

where $C_{1}: \mathbb{R} \rightarrow \mathbb{R}$ is an arbitrary smooth function.

Again in our case, $\partial \Psi / \partial x_{1}=0$ and $\Psi$ depends on $x_{2}$ only. Since $\partial \Psi / \partial x_{2}$ $=-f_{2} \theta_{2}(2+\Psi)$,

$$
\Psi\left(x_{1}, x_{2}\right)=C_{2} \cdot e^{-c \int_{0}^{x_{2}} f_{2}(t) d t}-2,
$$

where $C_{2} \in \mathbb{R}$.

The situation is almost symmetric when $\theta_{2}=0$ while $\theta_{1}=c \neq 0$.

Therefore, we have the following.

Proposition 2. For an arbitrary constant $c$, the family of all immersed surfaces $S=\iota\left(\mathbb{R}^{2}\right)$ in $\mathbb{R}^{3}$ with constant conformal principal curvatures 0 and $c$ is nonempty and parametrized by triples $\left(f_{2}, C_{1}, C_{2}\right)$, where $f_{2}, C_{1}$ are smooth real functions of one real variable and $C_{2}$ is a real number; $f_{1}$ and $C_{1}$ are either everywhere positive or everywhere negative. The corresponding surface has conformally invariant 1 -forms $\omega_{i}=f_{i} d x_{i}$ with $f_{1}$ given by (6), and the scalar conformal invariant $\Psi$ given by $(7)$. 
Assume now that, in addition to $\theta_{1}=0$ and $\theta_{2}=c \neq 0$, also $\Psi$ is constant. Then, by formula (7), $\Psi=-2$. Surfaces characterized by such conformal invariants are conformal images of loxodromic cylinders given, in Cartesian coordinates $\left(x_{1}, x_{2}, x_{3},\right)$ in $\mathbb{R}^{3}$, by the following equations:

$$
x_{1}=e^{c s / 4} \cos s, \quad x_{2}=e^{c s / 4} \sin s, \quad x_{3}=t,
$$

where $s, t \in \mathbb{R}$.

3. CCI-foliations. As mentioned in the introduction, a codimensionone foliation $\mathcal{F}$ of a manifold $M$ of constant sectional curvature is called a CCI-foliation whenever all its leaves have constant principal conformal curvatures $\theta_{1}, \theta_{2}$ and constant invariant $\Psi$.

Let us recall (see [CC, Vol. I, pp. 115-118]) that the limit set $\lim L$ of a leaf $L$ of a foliation $\mathcal{F}$ on a compact manifold $M$ is defined as the intersection of the closures in $M$ of the sets $L \backslash K$, where $K$ ranges over all compact subsets of $L$. If $L$ is noncompact, $\lim L$ is nonempty, compact and saturated. Recall also that a Reeb component of a foliation $\mathcal{F}$ on a 3-dimensional manifold $M$ is the product $R=S^{1} \times D^{2}, D^{2}$ being a closed 2-disc, such that the boundary $\partial R=T^{2}$ is a leaf while the interior $R_{0}$ of $R$ is foliated by planes $\mathbb{R}^{2}$ in such a way that $\lim L=\partial R$ for any leaf $L \subset R_{0}$. By Novikov's theorem ([No], see also [CC, Vol. II, Chapter 9]), any 2-dimensional foliation of the sphere $S^{3}$ contains a Reeb component. A foliation without Reeb components is said to be Reebless.

Theorem 1. Any CCI-foliation is Reebless.

Proof. Suppose that $R$ is a Reeb component of a CCI-foliation $\mathcal{F}$. Its interior $R_{0}$ contains no Dupin cyclides. Indeed, a Dupin cyclide $L$ has the so-called spherical two-piece property (STPP): any sphere $\Sigma$ separates $L$ into at most two components. On the other hand, any small sphere $\Sigma$ centered at a point $x_{0} \in \partial R$ separates any leaf $L \subset R_{0}$ into infinitely many components. Moreover, $R_{0}$ cannot contain other CCI-leaves. Indeed, the $\operatorname{limit} \operatorname{set} \lim L$ of a cylinder over a logarithmic spiral consists of a single line, and the limit set of its conformal image coincides with either a single line or a single circle and cannot fill all the boundary $\partial R$.

Corollary 1. A compact 3-dimensional manifold $M$ of constant curvature 1 admits no CCI-foliations.

Proof. If $\mathcal{F}$ were a CCI-foliation of $M$, then $\mathcal{F}$ would lift to a CCIfoliation $\widetilde{\mathcal{F}}$ of the universal cover $\widetilde{M}=S^{3}$ which-by Novikov's theoremwould contain a Reeb component. A contradiction.

Now, let us turn to compact hyperbolic 3-manifolds of curvature -1 . 
Assume that $M$ is such a manifold equipped with a 2-dimensional CCIfoliation $\mathcal{F}$. Lift $\mathcal{F}$ to $\widetilde{M}=H^{3}$, the universal cover of $M$. Denote by $\widetilde{\mathcal{F}}$ the lift of $\mathcal{F}$. Clearly, $\widetilde{\mathcal{F}}$ is a CCI-foliation of $\widetilde{M}$ by surfaces which are connected components of intersections $S \cap B, S$ being a complete CCI-surface in $\mathbb{R}^{3}$ and $B$ being the unit 3-ball representing $\widetilde{M}$. Our classification of CCI-surfaces shows that a priori a leaf $\widetilde{L}$ of $\widetilde{\mathcal{F}}$ can be (topologically) (1) a torus, (2) a torus with a disc (or a point) removed, (3) a cylinder, or (4) a disc.

We are going to show that all of them are discs. By Theorem 1, tori cannot occur: any toral leaf would bound a solid torus containing a Reeb component. Cases (2) and (3) can be treated simultaneously: a torus without a disc becomes a cylinder after intersecting with a smaller ball $B^{\prime}$ contained in $B$. Without loss of generality, we may assume that the sphere $\Sigma^{\prime}=\partial B^{\prime}$ intersects our cylindrical leaf $\widetilde{L}$ transversely and bounds (together with $\widetilde{L}$ ) a solid cylinder $C$ whose boundary consists of $\widetilde{L}$ and two discs $D_{1}$ and $D_{2}$ contained in $\Sigma^{\prime}$. By arguments of the proof of Theorem 6.3.1 in [LW], not all the leaves in $C$ are Dupin, therefore some of them coincide with the connected components of $S \cap B, S$ being a loxodromic cylinder. However, any such component intersects the boundary $\partial C$ transversely at some points of $\widetilde{L}$, a contradiction. Consequently, all the leaves of $\widetilde{\mathcal{F}}$ are topological open discs.

The boundaries of those discs are contained in the sphere $\Sigma(\infty)$, the ideal boundary of $\widetilde{M}$. Denote by $\widetilde{\mathcal{F}}(\infty)$ the union in $\Sigma(\infty)$ of all those boundaries.

Our manifold $M$ is of the form $M=\widetilde{M} / \Gamma$ for a discrete group $\Gamma$ of isometries of the hyperbolic 3 -space. The group $\Gamma$ acts on $\Sigma(\infty)$, and since $M$ is compact, this action is minimal, that is, the orbits $\Gamma(\zeta)$ of all points $\zeta \in \Sigma(\infty)$ are dense. Since $\widetilde{\mathcal{F}}$ is the lift of a foliation of $M$, the set $\widetilde{\mathcal{F}}(\infty)$ itself and its complement $\widetilde{\mathcal{F}}(\infty)^{c}$ in $\Sigma(\infty)$ are $\Gamma$-invariant, therefore dense if nonempty. The same observation applies to the open set $\widetilde{\mathcal{F}}(\infty)^{\dagger}$ of all points of $\widetilde{\mathcal{F}}(\infty)$ where the CCI-surface containing a leaf intersects $\Sigma(\infty)$ transversely.

If $\widetilde{\mathcal{F}}$ contains a piece of a loxodromic cylinder, the set $\widetilde{\mathcal{F}}(\infty)^{\dagger}$ is nonempty. So is its complement $\left(\widetilde{\mathcal{F}}(\infty)^{\dagger}\right)^{c}$ : otherwise, $\widetilde{\mathcal{F}}$ would determine a regular 1dimensional foliation of the sphere $\Sigma(\infty)$. Therefore, $\left(\widetilde{\mathcal{F}}(\infty)^{\dagger}\right)^{c}$ is dense, and consequently intersects its own complement $\widetilde{\mathcal{F}}(\infty)^{\dagger}$, a contradiction.

If $\widetilde{\mathcal{F}}$ contains no pieces of loxodromic cylinders, it becomes a Dupin foliation and we arrive at a contradiction as in [LW].

The argument above proves the following.

TheOREM 2. A compact 3-dimensional manifold $M$ of constant curvature -1 admits no CCI-foliations. 
4. A final remark. So far, the authors have no examples of surfaces with constant conformal principal curvatures $\theta_{1}, \theta_{2}$ and nonconstant Bryant invariant $\Psi$. Examples of such surfaces would be of interest. Foliations with all leaves of constant conformal principal curvatures could be called $C C C$ foliations. Existence of such foliations on the 3-dimensional sphere and compact hyperbolic 3-manifolds seems to be of some interest.

If one could prove that, as in the case of CCI-foliations, there are no CCCfoliations either, the following question would appear: How much further would one need to weaken the geometrical conditions in order to obtain examples of foliations with all leaves satisfying the new conditions?

\section{References}

[Br] R. Bryant, A duality theorem for Willmore surfaces, J. Differential Geom. 20 (1984), 23-53.

[CSW] G. Cairns, R. Sharpe and L. Webb, Conformal invariants for curves and surfaces in three dimensional space forms, Rocky Mountain J. Math. 24 (1994), 933-959.

[CC] A. Candel and L. Conlon, Foliations I, II, Amer. Math. Soc., Providence 2000, 2003.

[Fi] A. Fialkov, Conformal differential geometry of a subspace, Trans. Amer. Math. Soc. 56 (1944), 309-433.

[LW] R. Langevin and P. Walczak, Conformal geometry of foliations, Geom. Dedicata 132 (2008), 135-178.

[No] P. Novikov, Topology of foliations, Trans. Moscow Math. Soc. 14 (1965), 268-305.

[Tr] A. Tresse, Sur les invariants différentiels d'une surface par rapport aux transformations conformes de l'espace, C. R. Acad. Sci. Paris 114 (1892), 948-950.

Katedra Geometrii

Wydział Matematyki i Informatyki

Uniwersytet Łódzki

90-238 Łódź, Poland

E-mail:mak@math.uni.lodz.pl

pawelwal@math.uni.lodz.pl
Instytut Matematyczny PAN 00-956 Warszawa, Poland

Received 4.2.2008

and in final form 1.3.2008

(1854) 